\title{
Extended Abstracts
}

\section{Table of Contents}

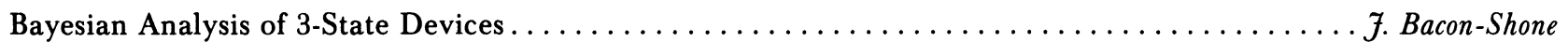

\section{Bayesian Analysis of 3-State Devices}

\section{J. Bacon-Shone, Member ASQC University of Hong Kong, Hong Kong}

Key Words - 3-State devices, Failure mode, Partial failure, Bayesian analysis.

There have been numerous papers detailing the use of Bayesian inference regarding the stochastic behaviour of 2state devices. This paper extends these methods to cover the behaviour of 3-state devices in equilibrium. The posterior distribution of the state probabilities is derived under two models:

1. Two types of $s$-independent failures.

2. Partial and complete failures.

The (constant) failure and repair rates are assumed to follow s-independent Gamma prior distributions and the devices are assumed to have Markov behaviour. It is shown that, up to a constant of proportionality, the posterior distribution has a simple form for both models. Estimation is discussed and, in particular, the consequences of a quadratic loss function are examined. Finally, the ideas are illustrated by means of a numerical example. Full details are available in a separately available Supplement.

\section{SUPPLEMENT}

NAPS document No. 03874-A; 8 pages in this Supplement. For current ordering information, see "Information for Readers \& Authors" in a current issue. Order NAPS document No. 03874, 26 pages. ASIS-NAPS; Microfiche Publications; POBox 3513, Grand Central Station; New York, NY 10017 USA.

\section{AUTHOR}

Dr. John Bacon-Shone; Department of Statistics; University of Hong Kong; Pokfulam Road; HONG KONG.

Manuscript TR80-168 received 1980 December 16; revised 1981 August 27.

\section{Availability of a 2-Unit Cold-Standby System with Degraded State}

\author{
Antonin Lesanovsky \\ Mathematical Institute of the Czech. Acad. \\ of Science, Prague
}

Key Words - Standby redundancy, Point availability, Laplace transform, Laplace-Stieltjes transform.

A cold standby redundant system with two i.i.d. units and one repair facility is studied. Units can be in three states: good, degraded, and failed. We suppose that only the following state-transitions of a unit are possible: good goes to degraded, degraded goes to failed, degraded goes to good, failed goes to good. Transition times of a unit between the states good, degraded, and failed are i.i.d. random variables. The repair of a degraded unit causes it to become good. This can be interpreted as a preventive maintenance, the realization of which depends on states of both units. Three conditions on the starting state of the system are considered. Point available of the system is derived by means of the theory of regenerative processes and is expressed in terms of Laplace and Laplace-Stieltjes transforms. For derivations and results, see the separately available Supplement.

\section{SUPPLEMENT}

NAPS document No. 03877-A; 8 pages in this Supplement. For current ordering information, see Information for Readers and Authors in a current issue. Order NAPS document No. 03877, 19 pages. ASIS-NAPS; Microfiche Publications; POBox 3513, Grand Central Station; New York, NY 10017 USA.

\section{AUTHOR}

Dr. Antonin Lesanovsky; Mathematical Institute of Czech. Acad. Sci.; Zitna 25; 11567 Praha 1 CZECHOSLOVAKIA.

Manuscript TR81-06 received 1981 January 24; revised 1981 October 10. 